\title{
The maternal JAK/STAT pathway of Drosophila regulates embryonic dorsal-ventral patterning
}

E.S.S. Lopes and H.M. Araujo
Departamento de Histologia e Embriologia, Universidade Federal do Rio de Janeiro, Rio de Janeiro, RJ, Brasil

\section{Correspondence \\ H.M. Araujo \\ Departamento de Histologia e \\ Embriologia, UFRJ \\ CCS, BI. F, Sala F2-031 \\ Av. Brig. Trompowski, s/n \\ 21949-900 Rio de Janeiro, RJ \\ Brasil \\ E-mail: haraujo@histo.ufrj.br}

Presented at the $\mathrm{XI}$ Congresso Brasileiro de Biologia Celular, Campinas, SP, Brazil, July 15-18, 2004.

Research supported by FAPERJ and FIRCA/NIH TW001329-03 to $\mathrm{H}$. Araujo, and a CNPq fellowship to E.S.S. Lopes.

Received June 1, 2004

Accepted September 23, 2004

\begin{abstract}
Activation of NFkB plays a pivotal role in many cellular processes such as inflammation, proliferation and apoptosis. In Drosophila, nuclear translocation of the NFKB-related transcription factor Dorsal is spatially regulated in order to subdivide the embryo into three primary dorsal-ventral (DV) domains: the ventral presumptive mesoderm, the lateral neuroectoderm and the dorsal ectoderm. Ventral activation of the Toll receptor induces degradation of the IкB-related inhibitor Cactus, liberating Dorsal for nuclear translocation. In addition, other pathways have been suggested to regulate Dorsal. Signaling through the maternal BMP member Decapentaplegic (Dpp) inhibits Dorsal translocation along a pathway parallel to and independent of Toll. In the present study, we show for the first time that the maternal JAK/STAT pathway also regulates embryonic DV patterning. Null alleles of loci coding for elements of the JAK/STAT pathway, hopscotch (hop), marelle ( $m r l$ ) and zimp (zimp), modify zygotic expression along the DV axis. Genetic analysis suggests that the JAK kinase Hop, most similar to vertebrate JAK2, may modify signals downstream of Dpp. In addition, an activated form of Hop results in increased levels of Cactus and Dorsal proteins, modifying the Dorsal/ Cactus ratio and consequently DV patterning. These results indicate that different maternal signals mediated by the Toll, BMP and JAK/ STAT pathways may converge to regulate NFkB activity in Drosophila.
\end{abstract}

Key words

- JAK kinase

- Drosophila

- Embryonic patterning

- hopscotch

- NFKB

\section{Introduction}

The establishment of the dorsal-ventral (DV) axis of the Drosophila embryo depends on processes acting during both oogenesis and embryogenesis. DV asymmetry, first generated during oogenesis, is ultimately transmitted to the embryo in the form of a nuclear gradient of the NFKB-related transcription factor Dorsal. A series of maternally transcribed genes exert their effects during early embryogenesis (1). Several of these genes encode components of a proteolytic cascade leading to the production of the activated ligand Spätzle (2-5), which binds to and activates the Toll receptor on the ventral side of the embryo. Genes functioning downstream of Toll encode cytoplasmic components that convey this spatial information to the embryo. The final steps in this intracellular machinery involve phosphorylation, ubiquitination and degradation of the I $\mathrm{KB}$ 
homologue Cactus, releasing Dorsal for nuclear translocation (see references in Ref. 1). A ventral to dorsal nuclear gradient of Dorsal protein is thus formed, leading to the establishment of the three primary domains of the embryo: the ventral presumptive mesoderm, the lateral neuroectoderm and the dorsal ectoderm (6-12).

More recently, it has been shown that signaling through the tumor growth factor- $\beta$ (TGFB) family member decapentaplegic (dpp) also modifies the Dorsal gradient (13). Increased signaling through maternal $d p p$, the Drosophila BMP2/4 orthologue (14), induces a shift towards more dorsal fates in the embryo. Conversely, a decrease in the maternal dose of the BMP antagonist short gastrulation (sog; 11,15) induces a similar phenotype. It has been suggested that maternal $d p p$ modifies the Dorsal gradient by regulating Cactus degradation through a Toll-independent pathway. The present model proposes that Dpp inhibits Cactus degradation, retaining Dorsal in the cytoplasm (13). The exact contribution of the signalindependent versus signal-dependent pathways of Cactus degradation to the establishment of the Dorsal gradient remains to be elucidated. However, there are indications that the activity of other genes may converge to regulate Dorsal and Cactus, thus contributing to the establishment of the embryonic DV axis.

Many different signals may regulate proteins of the NFKB/c-rel family. For instance, it has been shown in Drosophila that Dorsal may be phosphorylated (16-18) and that ventral signals modify Dorsal to regulate nuclear import independent of Cactus (19). Conversely, Cactus is regulated through several mechanisms. As cited above, degradation of Cactus may follow through the Toll-dependent (20) or -independent pathways (21-23). In addition to phosphorylation regulated by ventral signals, Cactus may also be phosphorylated by casein kinase II (22). Actually, proteins of the I $\mathrm{B}$ family from vertebrates and invertebrates present several regulatory modules which are targeted by phosphorylation.
In an attempt to identify other molecules that regulate maternal $\mathrm{NF \kappa B} / \mathrm{I \kappa B}$ signaling in Drosophila we have undertaken a directed screening for elements that modify a dorsal sensitized background. As a result of this analysis, we present evidence that elements of the JAK/STAT pathway also contribute to the establishment of the embryonic DV axis. Genetic data indicate that elements of this pathway may function by non-classical mechanisms to modify the Dorsal gradient. In addition, our results suggest that Hopscotch, most similar to vertebrate Janus kinase JAK2 (24), regulates the total levels of both Cactus and Dorsal proteins.

\section{Material and Methods}

\section{Fly stocks}

Canton S (CS) was used as the wild type. All mutants, balancers and chromosomal markers used in this study were obtained from the Bloomington Indiana Stock Center, Bloomington, IN, USA. All embryos analyzed in this study resulted from crosses between the maternal genotypes listed with male CS. For activation of the temperaturesensitive hop[Tum] allele females and resulting embryos were kept at $29^{\circ} \mathrm{C}$ until embryo collection.

\section{In situ hybridization}

In situ hybridization was performed as described previously with antisense RNA probes (25) using full-length cDNAs as template.

\section{Immunoblot analysis}

For analysis of Cactus, Dorsal and Tubulin, protein extracts, SDS-PAGE and immunoblots were performed as described (13). For quantitation of immunoblot bands from autoradiograms, several exposures were quantitated using the Histogram function of 
Photoshop, by determining the intensity of white in negative images. To determine the ratio of Dorsal/Cactus, relative protein amounts were initially determined by dividing the intensity of Cactus or Dorsal autoradiographic bands by the corresponding Tubulin bands to obtain CacT and DIT. The values obtained were then used to define the normalized DIT/CacT used as the Dorsal/ Cactus ratio.

\section{Results}

\section{Elements of the JAK/STAT pathway interact with Dorsal}

DV territories in the Drosophila blastoderm embryo can be visualized by in situ hybridization with specific RNA probes. In wild-type embryos, probes against the ventral nervous system defective ( $v$ nd) gene reveal expression in the ventral-most third of the lateral neuroectoderm (8). The pattern is highly reproducible and forms a precise border with the ventral presumptive mesoderm $(8,13)$ (Figure 1A). However, in embryos laid by mothers whose dose of the dorsal $(d l)$ gene was reduced to half $(d l-/+)$ this border is not as precise, especially in an anterior domain where the head fold will later form (13). In a small percentage of these embryos (3\%; Table 1) vnd expression totally invades the ventral territory at $30 \%$ egg length. Dorsal is sensitive to gene dosage (26-28), and alterations in the dose of genes that interact with the Dorsal pathway modify the penetrance or expressivity of the $d l-/+$ phenotype (13). Therefore, we have used the maternal $d l-/+$ as our "sensitized background" to search for other genes that interact with the Dorsal pathway.

We have screened for elements of other pathways that have been shown to interact genetically or biochemically with $\mathrm{NF \kappa B} / \mathrm{I} \kappa \mathrm{B}$ in vertebrates or invertebrates. Null or hypomorphic mutants for elements of the IL-1, IL-2 and calcium signaling pathways were crossed to a $d l$ mutant line and the embryos from mothers double heterozygous for these alleles were collected for in situ analysis (data not shown). Amongst the loci analyzed, elements of the Drosophila JAK/STAT pathway consistently modified the $d l-/+$ phenotype. Embryos derived from mothers double heterozygous for a null hopscotch allele (hop [2]) and a null $d l$ allele $(d l[15])$ presented an increased penetrance of the $d l-/+$ phenotype (30\%; Figure 1B and Table 1). A similar effect could be observed with mutant alleles of downstream elements of the JAK/STAT pathway such as a null allele of the Stat3/Stat5 homologue marelle $(\mathrm{mrl})$ (24) and the PIAS negative regulator zimp (zimp; Table 1; 29). It may seem surprising that all three elements increase the penetrance
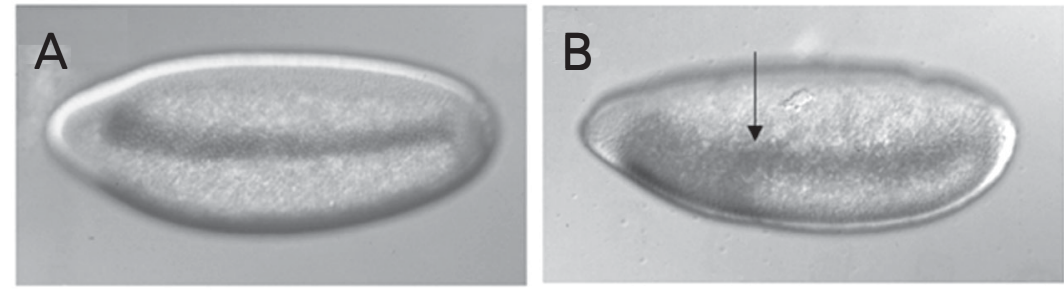

Figure 1. hopscotch regulates zygotic gene expression along the dorsal-ventral axis. Blastoderm stage embryos collected from wild-type (A) or hop[2]; dI/[15]/+ (B) mothers and processed for in situ hybridization with a vnd probe. Observe the ventral invasion of vnd expression in B (arrow). Anterior is left, dorsal is up.

Table 1. Elements of the maternal JAK/STAT pathway alter embryonic gene expression along the dorsal-ventral axis.

Maternal genotype Penetrance of phenotype, \% (N)

Wild type

$d /[15] /+$

0 (122)

hop $[2] /+; d l-/+$ 3*

dl[15]/+; mrl[06346]/+

d/[15]/zimp[03697]

$\mathrm{Dp}(d p p) /+; d /[15] /+$

hop[2]/Dp(dpp); dl[15]/+

$35(40)$

$26(50)$

21 (19)

52 (101)

$\mathrm{Dp}(d p p) /+; d /[15] /+; m r /[06346] /+$

$70(83)$

$16(31)$

$\mathrm{Dp}(d p p) /+; d /[15] / z i m p[03697]$

Embryos were collected from the maternal genotypes listed and processed for in situ hybridization with a vnd probe. The penetrance of the dorsalized embryonic phenotype revealed by ventral invasion of vnd expression (as shown in Figure 1B) is presented. Penetrance is defined as the percentage of embryos that present the phenotype. The total number of embryos analyzed for each condition is shown in parentheses. * Data taken from Ref. 13 
of the $d l-/+$ phenotype, especially considering that Zimp inhibits signaling through Hop and Mrl. However, it should be kept in mind that this type of experiment, which relies on disturbance of the balance between different regulatory elements, does not necessarily reveal the direction of scored interactions.

We have shown that the Dpp pathway interacts with Dorsal. Alterations in the dose of maternal $d p p$ by itself does not seem to modify the embryonic DV axis (13). However, embryos derived from mothers containing a duplication of the $d p p$ locus $(\mathrm{Dp}(d p p))$ and heterozygous for a null $d l$ allele show a great increase in the penetrance of the $d l-/+$ phenotype (Table 1) (13). In order to determine whether elements of the JAK/STAT pathway could modify the $\mathrm{Dp}(d p p) /+; d l-/+$ phenotype we generated embryos from mothers triple heterozygous for $\mathrm{Dp}(d p p), d l$ - and alleles of hop, $\mathrm{mrl}$ or zimp. Interestingly, we could observe an additive effect of hop and $\operatorname{Dp}(d p p)$ on the embryonic phenotype, while alleles of $\mathrm{mrl}$ and zimp decreased the penetrance of the $\operatorname{Dp}(d p p) /+; d l-/+$ phenotype (Table 1$)$. Again, these experiments did not permit us to reach any conclusions regarding the direction of the interactions scored. However, they did suggest some dissociation between the effects generated by hop versus $m r l$ and zimp.

Table 2. The effects of maternal $d p p$ and hop on embryonic gene expression are not additive.

\begin{tabular}{lccccc} 
Maternal genotype & $\begin{array}{c}\text { Penetrance of } \\
\text { phenotype, \% (N) }\end{array}$ & \multicolumn{4}{c}{$\begin{array}{c}\text { Expressivity (\% egg length with } \\
\text { ventral invasion of vnd expression) }\end{array}$} \\
\cline { 3 - 7 } & & 0 & 30 & 50 & 90 \\
\hline Wild type & $0(115)$ & 100 & - & - & - \\
hop [Tum]/+ & $0(45)$ & 100 & - & - & - \\
hop[Tum]/+; dl[15]/+ & $84.0(50)$ & 16.0 & 34.0 & 14.0 & 36.0 \\
Dp(dpp)/+; d/[15]/+ & $66.7(66)$ & 33.3 & 40.9 & 6.1 & 19.7 \\
Dp(dpp)/hop[Tum]; dl[15]/+ & $80.5(41)$ & 19.5 & 34.2 & 14.6 & 31.7 \\
\hline
\end{tabular}

Blastoderm stage embryos collected from the maternal genotypes listed were processed for in situ hybridization with a vnd probe. Phenotype and expressivity classes correspond to those shown in Figure 2. The total number of embryos analyzed for each condition is shown in parentheses.
Using an activated hop allele to understand the mechanism of Hop action

In order to further test the relationship between the JAK/STAT and Dpp signaling pathways in the context of DV patterning, we used a temperature-sensitive gain-of-function hop allele that is independent of upstream receptor activation. This allele, termed hop [Tum] (for Tumoral), generates a hyperactivated JAK kinase at $29^{\circ} \mathrm{C}(30,31)$. We intended to test if hop[Tum] could block a dorsalizing Dpp signal generated by an increase in the dose of $d p p$. Surprisingly, though, activation of signaling through hop by using the hop [Tum] allele also increased the $d l-/+$ phenotype (Table 2), i.e., it induced a dorsalized phenotype. As a dominant negative form of hop was not available and as null hop clones would effect earlier development of follicles (32), we tested whether $h o p$ [Tum] and a duplication of $d p p$ would present additive dorsalizing effects, suggesting that they alter DV patterning through different pathways.

At $29^{\circ} \mathrm{C}$ mothers double heterozygous for hop [Tum] and $d l$ - generated $84 \%$ of dorsalized embryos, while mothers double heterozygous for $\mathrm{Dp}(d p p)$ and $d l$ - generated $67 \%$ of dorsalized embryos (Table 2). Mothers triple heterozygous for hop [Tum], $\operatorname{Dp}(d p p)$ and $d l$ - generated $80 \%$ of embryos with invasion of $v$ nd expression in the ventral domain (Table 2, Figure 2). The penetrance of the phenotypes for the triple heterozygotes could represent either average or equivalent effects of $h o p$ [Tum] and $\mathrm{Dp}(d p p)$ on DV patterning. Unfortunately, low viability of the maternal genotypes resulted in a modest number of embryos analyzed, precluding the use of statistical tests. Nevertheless, our results argue against an additive effect of hop[Tum] and $\mathrm{Dp}(d p p)$. By analyzing the expressivity of the phenotypes for the above conditions, this can be seen more clearly (Figure 2, Table 2). While increasing the dose of $d p p$ in $d l-/+$ mothers induced 
ventral vnd invasion mostly in the anterior part of the embryo, inducing hop activity with or without a duplication of $d p p$ extended the ventral invasion of vnd expression to more posterior regions of the embryos (Figure 2, Table 2). The distribution among expressivity classes was similar between hop [Tum]/+; dl-/+ and hop[Tum]/ $\mathrm{Dp}(d p p) ; d l-/+$.

These results indicate that, with activation of the Hop kinase, Dpp can exert no further effect on DV patterning of the embryo. This would suggest that Dpp either acts directly to regulate Hop activity or requires Hop to regulate some downstream element of the maternal Dpp pathway.

\section{Activated Hop modifies Cactus and Dorsal protein levels}

Formation of the Dorsal gradient depends on a precise balance of Cactus and Dorsal protein levels. In addition to regulation of
Cactus degradation through the Toll-dependent and -independent pathways, the level of Cactus protein depends on the amount of Dorsal protein. It has been shown that genetically modifying the dose of $d l$, and thus the amount of Dorsal protein, results in a concomitant alteration in the total level of Cactus (28). Therefore, one possible mechanism for hop action would be to regulate the levels of Dorsal and Cactus. In a wild-type background, hop[Tum] does not alter the level of either protein (Figure 3A,B, lane 2). On the other hand, hop [Tum] induced an increase in the levels of both Cactus and Dorsal in a $d l-/+$ background (Figure 3A,B, lanes 3 and 4). Measurement of the protein band intensities indicated that the Cactus to Dorsal ratio was increased above that of the wild type (Figure 3, Legend), suggesting that the ventral invasion of vnd expression in embryos from $h o p /+; d l /+$ mothers is a result of higher retention of Dorsal in the cytoplasm by increased Cactus levels.
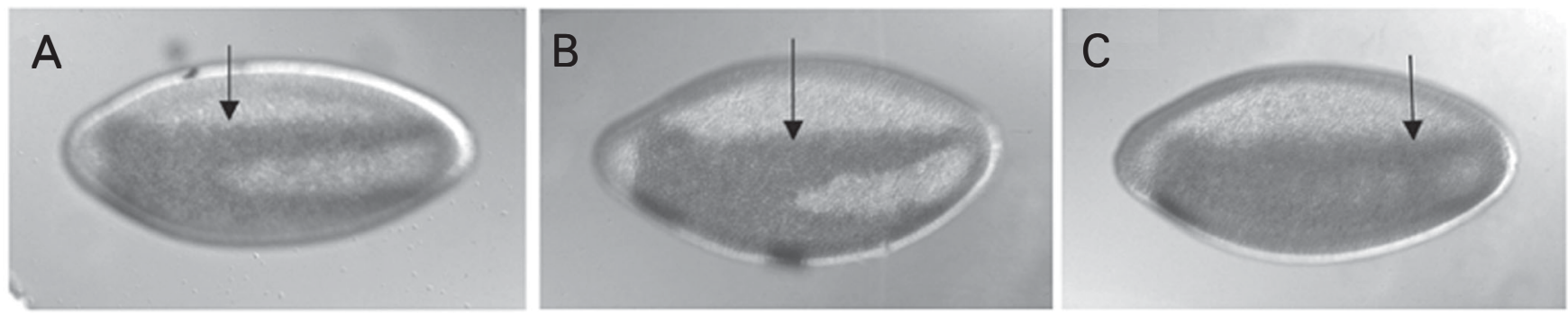

Figure 2. Embryonic phenotypes generated by activated Hop are variable. Blastoderm stage embryos were collected from hop[Tum]; dl[15]/+ mothers and processed for in situ hybridization with a vnd probe. At $29^{\circ} \mathrm{C}$ the expressivity of the phenotype analyzed is variable, with vnd expression invading the ventral domain (arrows) at 30\% (A), 50\% (B) or 90\% (C) egg length, with egg length determined from the anterior to the posterior end of the embryo. No invasion is defined as $0 \%$. Anterior is left, dorsal is up.

A

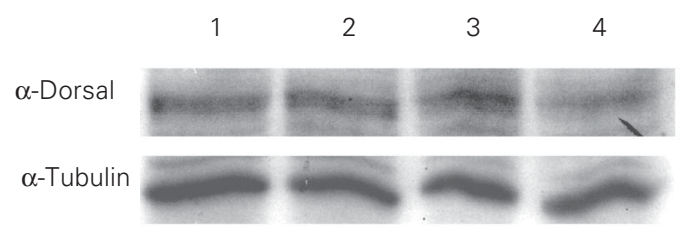

B

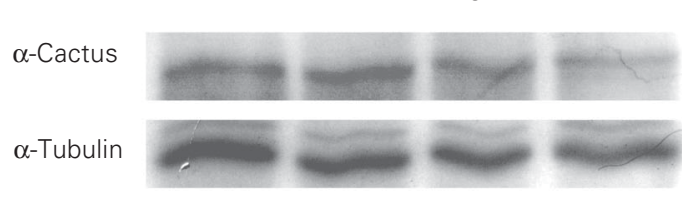

Figure 3. Activated hop increases the levels of Dorsal and Cactus proteins. Total embryonic extracts were prepared from 0- to 1-h pre-blastoderm embryos, run on $8 \%$ SDS-PAGE and probed with antibodies against Dorsal (A) or Cactus (B). The same blot was subsequently probed for anti-Tubulin (lower panels) to assure that equivalent amounts of protein were loaded for each sample. Embryos were collected from wild-type (lane 1), hop[Tum]/ + (lane 2); hop[Tum]/+; d/[15]/+ (lane 3) or d/[15]/+ (lane 4) mothers. The relative amounts of Dorsal/Cactus were: 1.19 for wild type, 1.18 for $d /[15] /+$, and 0.92 for hop $[$ Tum]/+; d/[15]/+. 


\section{Discussion}

\section{hopscotch may signal through two different pathways to regulate embryonic DV patterning}

The Drosophila JAK/STAT pathway plays several roles during development. These include segmentation, eye development, hematopoiesis and gametogenesis (see references in Ref. 33). In addition, an important role in the Drosophila immune response and blood cell proliferation has been described (reviewed in Ref. 34). Interestingly, this may be an evolutionarily conserved role as vertebrate JAK/STAT is fundamental in regulating proliferation and differentiation of hematopoietic stem cells and in the immune response (35). We describe here for the first time a role for JAK/STAT signaling in embryonic DV patterning.

All elements of the JAK/STAT pathway tested, hop, $m r l$ and zimp, somehow altered vnd expression, indicating that classical elements of the JAK/STAT pathway regulate embryonic DV patterning. However, while all three loci increased the penetrance of the $d l-/+$ phenotype, only hop could still induce an increase in this penetrance in the presence of a duplication of $d p p$. Even though these genetic tests do not reveal the direction of interactions, it is striking that hop versus $m r l$ and zimp showed diverging effects only in the presence of Dpp. This could suggest that, in addition to hop acting on a classical pathway to activate $m r l$, it could also act independently on a pathway more related to $d p p$. For instance, Hop may be required to phosphorylate some element of the signal-independent pathway of Cactus degradation, downstream of Dpp. It has been shown that the vertebrate JAK2 kinase is able to phosphorylate I $\mathrm{KB}$ in response to erythropoietin (36). This results in increased degradation of $\mathrm{I} \kappa \mathrm{B}$, with a consequent protection from apoptosis. Interestingly, increased Hop activity attained by using the temperature-sensi- tive hop[Tum] allele increases the total amount of Cactus protein, a fact possibly due to altered degradation. Furthermore, Dpp also inhibits Cactus degradation (13). Finally, $\mathrm{Dp}(d p p)$ does not increase the penetrance of the DV phenotype induced by hop [Tum], indicating that Hop activity is required downstream of Dpp to regulate Cactus levels. It should be pointed out, however, that our data do not address whether Cactus is a direct target of Hop, or whether it regulates the degradation of Cactus protein.

Other evidence that hop may exert an effect on DV patterning independently of $m r l$ is the fact that hop [Tum] altered the levels of Cactus protein. It seems unlikely that $\mathrm{mrl}$ regulates the level of expression of cactus mRNA, especially if we consider that cactus is maternally transcribed and that no zygotic expression at the early embryonic stages has been described (16). Alternatively, $m r l$ could regulate the stability of cactus mRNA or protein.

Even though Hop may act independently of $\mathrm{Mrl}$, it also needs to be invoked to explain the effect of $m r l$ on DV patterning. As a transcription factor, STAT92E, encoded by $m r l$, must be activated by a JAK kinase to enter the nucleus and bind to regulatory elements of target genes (24). Subsequently, $\mathrm{mrl}$ may regulate the expression of zygotic genes, such as vnd, which are targets of regulation by $d l$. An in silico screen for $m r l$ and $d l$ binding sites in common regulatory regions of $d l$ target genes may help elucidate this point. Other possible points of regulation by $\mathrm{mrl}$ would be regulation of Dorsal nuclear translocation independent of Cactus and modulation of other elements of the Toll pathway, all of which lead to alterations of the embryonic DV axis. Whatever the mechanisms of action of Hop and Mrl that may be revealed in the future, in view of the above considerations, it seems plausible that Hop acts on two points: activating Mrl and modifying Cactus levels independently of Mrl. However, additional analysis will be 
necessary to test this prediction.

\section{The role of the JAK/STAT pathway in defining DV territories}

Intersection between different signal transduction pathways results in a combinatorial control that may help fine-tune and integrate functional responses. Intersection between interleukin and NFKB pathways has been described in the regulation of nitric oxide synthase transcription (37), and of the type 2 Toll-like receptor (38). Intercommunication between interleukin and the TGF- $\beta$ pathway has also been reported. For instance, TGF- $\beta$ regulates the transcription of interleukin genes (39), and has an important role during the immune response. Another level of intersection between these elements may take part in the cytoplasmic environment as described during neuroprotection in vertebrates (36) and as suggested in the present study.

Our results suggest intersection between the Drosophila JAK/STAT, NFKB and TGF$B$ family pathway elements. In spite of the clear genetic interactions between hop, $d l$ and $d p p$, by examination of embryos derived from mothers with altered Hop signaling alone, we were unable to detect any effect on expression patterns along the DV axis (Lopes E, unpublished results). This leads us to ask what role hop may play in DV patterning. Actually, it is quite hard to understand why mothers heterozygous for hop (2), hop [Tum] or $\mathrm{Dp}(d p p)$ had no effect on DV patterning by themselves, while they did so when the dose of $d l$ was initially disturbed. One explanation would be that the Toll pathway is robust, accounting for subdivision of $\mathrm{DV}$ territories and their exact placement along the DV axis (40). Other pathways would have been co-opted to ensure that proper DV patterning takes place when direct signaling through Toll is disturbed. Certainly more experimentation will be necessary to test this hypothesis. It will also be very interesting to test the generality of the interactions described here in other model systems, and to assay the respective contributions of each of the interacting genes.

\section{Acknowledgments}

We are grateful to Silvania Nunes for fly food preparation and to the Bloomington Stock Center, Bloomington, IN, USA, for fly lines. We would also like to thank Dr. Ethan Bier (UCSD) for helpful discussions and intellectual support.

\section{References}

1. Morisato D \& Anderson KV (1995). Signaling pathways that establish the dorsal-ventral pattern of the Drosophila embryo. Annual Review of Genetics, 29: 371-399.

2. Schneider DS, Jin Y, Morisato D \& Anderson KV (1994). A processed form of the Spatzle protein defines dorsal-ventral polarity in the Drosophila embryo. Development, 120: 1243-1250.

3. Stein D, Roth S, Vogelsang E \& Nusslein-Volhard C (1991). The polarity of the dorsoventral axis in the Drosophila embryo is defined by an extracellular signal. Cell, 65: 725-735.

4. Morisato D (2001). Spatzle regulates the shape of the Dorsal gradient in the Drosophila embryo. Development, 128: 2309-2319.

5. Morisato D \& Anderson KV (1994). The spatzle gene encodes a component of the extracellular signaling pathway establishing the dorsal-ventral pattern of the Drosophila embryo. Cell, 76: 677-688.

6. Steward R (1987). Dorsal, an embryonic polarity gene in Drosophila, is homologous to the vertebrate proto-oncogene, c-rel. Science, 238: 692-694
7. Thisse C, Perrin-Schmitt F, Stoetzel C \& Thisse B (1991). Sequencespecific transactivation of the Drosophila twist gene by the dorsal gene product. Cell, 65: 1191-1201.

8. Mellerick DM \& Nirenberg M (1995). Dorsal-ventral patterning genes restrict NK-2 homeobox gene expression to the ventral half of the central nervous system of Drosophila embryos. Developmental Biology, 171: 306-316.

9. Jiang J, Kosman D, Ip YT \& Levine M (1991). The dorsal morphogen gradient regulates the mesoderm determinant twist in early Drosophila embryos. Genes and Development, 5: 1881-1891.

10. Ip YT, Park RE, Kosman D, Bier E \& Levine M (1992). The dorsal gradient morphogen regulates stripes of rhomboid expression in the presumptive neuroectoderm of the Drosophila embryo. Genes and Development, 6: 1728-1739.

11. Francois V, Solloway M, O'Neill JW, Emery J \& Bier E (1994). Dorsal-ventral patterning of the Drosophila embryo depends on a putative negative growth factor encoded by the short gastrulation 
gene. Genes and Development, 8: 2602-2616.

12. Doyle HJ, Kraut R \& Levine M (1989). Spatial regulation of zerknullt: a dorsal-ventral patterning gene in Drosophila. Genes and Development, 3: 1518-1533.

13. Araujo $H$ \& Bier $E$ (2000). sog and dpp exert opposing maternal functions to modify toll signaling and pattern the dorsoventral axis of the Drosophila embryo. Development, 127: 3631-3644.

14. Padgett RW, St Johnston RD \& Gelbart WM (1987). A transcript from a Drosophila pattern gene predicts a protein homologous to the transforming growth factor-beta family. Nature, 325: 81-84.

15. Ferguson EL \& Anderson KV (1992). Localized enhancement and repression of the activity of the TGF-beta family member, decapentaplegic, is necessary for dorsal-ventral pattern formation in the Drosophila embryo. Development, 114: 583-597.

16. Whalen AM \& Steward R (1993). Dissociation of the dorsal-cactus complex and phosphorylation of the dorsal protein correlate with the nuclear localization of dorsal. Journal of Cell Biology, 123: 523534.

17. Gillespie SK \& Wasserman SA (1994). Dorsal, a Drosophila Rel-like protein, is phosphorylated upon activation of the transmembrane protein Toll. Molecular and Cellular Biology, 14: 3559-3568.

18. Drier EA, Huang LH \& Steward R (1999). Nuclear import of the Drosophila Rel protein Dorsal is regulated by phosphorylation. Genes and Development, 13: 556-568.

19. Drier EA, Govind S \& Steward R (2000). Cactus-independent regulation of Dorsal nuclear import by the ventral signal. Current Biology, 10: $23-26$

20. Wasserman SA (2000). Toll signaling: the enigma variations. Current Opinion in Genetics and Development, 10: 497-502.

21. Reach $M$, Galindo RL, Towb $P$, Allen JL, Karin M \& Wasserman SA (1996). A gradient of cactus protein degradation establishes dorsoventral polarity in the Drosophila embryo. Developmental Biology, 180: 353-364

22. Liu ZP, Galindo RL \& Wasserman SA (1997). A role for CKII phosphorylation of the cactus PEST domain in dorsoventral patterning of the Drosophila embryo. Genes and Development, 11: 3413-3422.

23. Belvin MP, Jin Y \& Anderson KV (1995). Cactus protein degradation mediates Drosophila dorsal-ventral signaling. Genes and Development, 9: 783-793.

24. Hou XS, Melnick MB \& Perrimon N (1996). Marelle acts downstream of the Drosophila HOP/JAK kinase and encodes a protein similar to the mammalian STATs. Cell, 84: 411-419.

25. O'Neill JW \& Bier E (1994). Double-label in situ hybridization using biotin and digoxigenin-tagged RNA probes. Biotechniques, 17: 870, 874-875.

26. Steward R (1989). Relocalization of the dorsal protein from the cyto- plasm to the nucleus correlates with its function. Cell, 59: 1179-1188.

27. Rushlow CA, Han K, Manley JL \& Levine M (1989). The graded distribution of the dorsal morphogen is initiated by selective nuclear transport in Drosophila. Cell, 59: 1165-1177.

28. Govind S, Brennan L \& Steward R (1993). Homeostatic balance between dorsal and cactus proteins in the Drosophila embryo. Development, 117: 135-148.

29. Betz A, Lampen N, Martinek S, Young MW \& Darnell Jr JE (2001). A Drosophila PIAS homologue negatively regulates stat92E. Proceedings of the National Academy of Sciences, USA, 98: 9563-9568.

30. Hanratty WP \& Dearolf CR (1993). The Drosophila tumorous-lethal hematopoietic oncogene is a dominant mutation in the hopscotch locus. Molecular and General Genetics, 238: 33-37.

31. Harrison DA, Binari R, Nahreini TS, Gilman M \& Perrimon N (1995). Activation of a Drosophila Janus kinase (JAK) causes hematopoietic neoplasia and developmental defects. EMBO Journal, 14: 28572865.

32. McGregor JR, Xi R \& Harrison DA (2002). JAK signaling is somatically required for follicle cell differentiation in Drosophila. Development, 129: 705-717.

33. Hombria JC \& Brown S (2002). The fertile field of Drosophila Jak/ STAT signalling. Current Biology, 12: R569-R575.

34. Mathey-Prevot B \& Perrimon N (1998). Mammalian and Drosophila blood: JAK of all trades? Cell, 92: 697-700.

35. Hou SX, Zheng Z, Chen X \& Perrimon N (2002). The Jak/STAT pathway in model organisms: emerging roles in cell movement. Developmental Cell, 3: 765-778.

36. Digicaylioglu M \& Lipton SA (2001). Erythropoietin-mediated neuroprotection involves cross-talk between Jak2 and NF-kappaB signalling cascades. Nature, 412: 641-647.

37. Ganster RW, Taylor BS, Shao L \& Geller DA (2001). Complex regulation of human inducible nitric oxide synthase gene transcription by Stat 1 and NF-kappa B. Proceedings of the National Academy of Sciences, USA, 98: 8638-8643.

38. Musikacharoen T, Matsuguchi T, Kikuchi T \& Yoshikai Y (2001). NFkappa B and STAT5 play important roles in the regulation of mouse Toll-like receptor 2 gene expression. Journal of Immunology, 166: 4516-4524.

39. Fargeas C, Wu CY, Nakajima T, Cox D, Nutman T \& Delespesse G (1992). Differential effect of transforming growth factor beta on the synthesis of Th1- and Th2-like lymphokines by human T lymphocytes. European Journal of Immunology, 22: 2173-2176.

40. Stathopoulos A \& Levine M (2002). Linear signaling in the TollDorsal pathway of Drosophila: activated Pelle kinase specifies all threshold outputs of gene expression while the bHLH protein Twist specifies a subset. Development, 129: 3411-3419. 